\title{
RELACIÓN DE LA ESQUIZOTIPIA PSICOMÉTRICA CON VARIABLES EMOCIONALES Y SOCIOAMBIENTALES
}

\author{
José A. Muela, AnA GARCÍA-LeÓn y M. Dolores JimÉneZ \\ Facultad de Psicología, Universidad de Jaén
}

\begin{abstract}
Resumen: La esquizotipia es una alteración de la personalidad caracterizada por presentar signos y síntomas semejantes (y de menor intensidad) a los de la esquizofrenia. Parece haber relaciones entre esquizotipia y variables emocionales y socioambientales que pueden contribuir en el papel de este trastorno como marcador de vulnerabilidad a la esquizofrenia. El objetivo del presente estudio fue indagar sobre las diferencias emocionales y socioambientales entre sujetos con puntuaciones extremas en el Oxford-Liverpool Inventory (O-LIFE) y sus subescalas. Participaron 100 estudiantes universitarios, divididos en grupos de alta o baja puntuación según el O-LIFE y/o algunas de sus subescalas. Los altos en esquizotípia psicométrica muestran mayores puntuaciones en depresión (BDI), ansiedad-estado (STAI-E), ansiedad-rasgo (STAI-R) y hostilidad (Ho) que los bajos. A partir del análisis de los datos tomados mediante la Entrevista Estructurada para la Evaluación de Variables Socioambientales, se encontró también que los sujetos altos en esquizotipia dan menos importancia a las relaciones familiares y éstas son peores, exhiben mayor cantidad de acontecimientos vitales y aprovechan peor las horas de estudio que los que obtienen puntuaciones bajas. Estos resultados se manifiestan en mayor medida cuando se tiene en cuenta la puntuación total en el OLIFE o cuando se compara a los sujetos altos y bajos en la subescala de No-Conformidad-Impulsiva de dicho instrumento.
\end{abstract}

Palabras clave: Esquizotipia psicométrica, alteraciones emocionales, variables socioambientales, O-LIFE.

Relationships between psychometric schizotypy, and emotional and socioenvironment variables

\begin{abstract}
The aim of this study was to explore emotional and socioenvironmental differences between subjects with extreme scores in psychometric schizotypy. One hundred university students participated in this study, divided in groups with high or low scores in the Oxford-Liverpool Inventory (O-LIFE) and/or some of its subscales. Subjects with high psychometric schizotypy scores showed higher scores in depression (BDI), state anxiety (STAI-S), trait anxiety (STAI-T) and hostility (Ho) than low psychometric schizotypy subjects. Analysis of the scores taken with the Structured Interview for the Evaluation of Socioenvironment Variables showed subjects with high scores in psychotypy to give less importance to family relationships, that are worse, show more vital events and are less efficient in the use of their time for study than low score subjects. These results mainly appear in the global score of O-LIFE and in the Impulsivity Nonconformity subscale.
\end{abstract}

Keywords: Psychometric schizotypy, emotional disorders, socioenvironment variables, O-LIFE.

\section{INTRODUCCIÓN}

La esquizotipia es una alteración de la personalidad que se enmarca dentro del espectro esquizofrénico. Los sujetos esquizotípicos presentan malestar, una reducida capacidad para

Recibido: 12 enero 2007; aceptado 20 agosto 2007.

Correspondencia: Departamento de Psicología, Universidad de Jaén, Campus de las Lagunillas s/n, 23071 Jaén. Correoe: angarcia@ujaen.es las relaciones personales (distanciamiento en las relaciones íntimas y vida social empobrecida), así como distorsiones cognoscitivas o perceptivas y un comportamiento excéntrico. Dichos síntomas comienzan al inicio de la edad adulta y se pueden observar en diversos contextos (American Psychiatric Association, 2002). Algunos autores creen que, siguiendo el continuo normal-patológico, puede observarse que, aun siendo la personalidad esquizotípica un trastorno grave, existen rasgos aislados de estos 
sujetos que son propios de una personalidad normal (Millon y Davis, 2006), siendo susceptible de evaluarse a través de procedimientos de autoinforme (Fernández-Montalvo y Echeburúa, 2006).

Las personas con puntuaciones altas en esquizotipia pueden ser consideradas de alto riesgo para el desarrollo futuro de la esquizofrenia. Por una parte, esto se basa en la evidencia de una similitud entre rasgos esquizotípicos y síntomas esquizofrénicos como desorganización, pobreza psicomotora o distorsión de la realidad (Martínez et al., 1999; Paíno et al., 1997). Por otra parte, se fundamenta en el hecho de que algunos familiares de sujetos esquizofrénicos, aunque nunca llegan a desarrollar la enfermedad, muestran características esquizotípicas de personalidad (Hodges, Byrne, Grant y Johnstone, 1999). De acuerdo con el modelo de vulnerabilidad-estrés (Becoña, 2006), los rasgos esquizotípicos y la psicosis esquizofrénica podrían constituir diferentes puntos de un continuo asociados con distintos grados de riesgo (Straube y Oades, 1992).

En esta línea, diversas investigaciones han pretendido identificar la relación entre la esquizotipia y otras variables emocionales y socioambientales, que puedan ser identificadas como posibles índices de vulnerabilidad a este y otros tipos de trastornos (Cabrera et al., 2005; Edo y Ballester, 2006), y que han sido ampliamente validadas como marcadores de vulnerabilidad hacia la esquizofrenia (Godoy, 1990). En relación con variables emocionales, para investigadores como Kendler (1985) y Meehl (1962), la ansiedad puede ser incluida como un rasgo bastante prominente en los esquizotípicos. En este sentido, Braunstein-Bercovitz (2000), con población normal universitaria que puntuaba alto en esquizotipia, encontró que estos sujetos mostraban altos niveles de ansiedad que interferían en su capacidad atencional.

Por otra parte, también se ha encontrado una relación positiva entre la esquizotipia y síntomas clínicos de depresión y ansiedad. Así, Lenzenweger, Cornblatt y Putnick (1991), en un estudio en el que utilizaron población universitaria normal, encontraron que los sujetos que puntuaban alto en esquizotipia tenían puntuaciones muy altas o grandes niveles de depresión y ansiedad. Igualmente, Wolfradt y Straube (1998), en una muestra de sujetos normales adolescentes, hallaron que los factores de esquizotipia y las medidas de depresión y ansiedad correlacionaban de manera significativa. Por último, en un estudio de Lewandowski et al. (2006), se ha encontrado una asociación positiva entre síntomas de depresión y ansiedad y síntomas positivos de la esquizotipia. En otro tipo de estudio (Godoy et al., 1995), realizado con distintos miembros de una familia, se constató que la ansiedad era un rasgo sobresaliente en los hijos normales de los enfermos esquizofrénicos; es decir, población de alto riesgo para sufrir este tipo de trastornos.

Un último tipo de variable emocional que se ha visto está relacionada con la esquizotipia es la hostilidad. De hecho, autores como Shean y Wais (2000), en un estudio sobre conducta interpersonal en sujetos normales con puntuaciones altas en esquizotipia y su impacto sobre los demás, encontraron que estos sujetos mostraban más hostilidad que los sujetos con baja esquizotipia. Hay autores como Eurelings-Bontekoe, Van der Slikke y Verschuur (1997) que piensan que aquellas personas que sufren trastornos de la personalidad tienden a ser hostiles, además de usar estrategias de afrontamiento pasivo. Las personas que muestran rasgos esquizotípicos tienden a no buscar apoyo social y a evitar las situaciones difíciles además de no expresar sus sentimientos (su ira). Para estos autores la hostilidad sería el mayor predictor independiente de patología de la personalidad.

En cuanto a las variables socioambientales, Cornblatt, Lenzenweger, Dworkin y Erlenmeyer-Kimling (1992) propusieron un modelo que puede servir como posible explicación de los problemas que estos individuos tienen a nivel interpersonal y que, por tanto, podría influir en su ambiente. Para estos autores, los sujetos con puntuaciones altas en esquizotipia tendrían poca habilidad para procesar de forma adecuada la información relacionada con indicadores de comunicación interpersonal, lo que provocaría interacciones sociales dificultosas y estresantes.

A este respecto, Burman, Mednick, Machón, Parnas y Schulsinger (1987) encontraron que las relaciones familiares eran percibidas como 
igualmente satisfactorias por parte de sujetos diagnosticados con esquizotipia y por parte de sujetos sin ningún diagnóstico de patología. Estos autores examinaron las relaciones familiares antes de la aparición de la psicopatología en una muestra de sujetos de alto riesgo para la esquizofrenia. Se basaban en la teoría de que sujetos con esquizofrenia y esquizotipia comparten una porción similar de predisposición genética a la esquizofrenia y son los factores ambientales los que determinan la aparición y desarrollo del síndrome esquizofrénico. Los resultados demostraron que las relaciones familiares, en la descendencia de alto riesgo que posteriormente desarrollaban la esquizofrenia, eran percibidas como significativamente menos satisfactorias que las relaciones familiares de los descendientes de alto riesgo que posteriormente eran diagnosticados con trastorno de personalidad esquizotípico o aquellos sin enfermedad mental. Estos hallazgos proporcionan un apoyo adicional al modelo de diátesis-estrés, sugiriendo que las personas con riesgo a la esquizofrenia están descompensadas en parte en su desarrollo, debido al estrés ambiental asociado con relaciones familiares insatisfactorias. Sin embargo, estos resultados no se corresponderían con lo esperado según el modelo anteriormente mencionado al no diferenciar entre la percepción de los sujetos esquizotípicos y la de los normales.

Para Waldeck y Miller (2000) las dificultades sociales en personas con esquizofrenia (debidas a que nunca podrán desarrollar las habilidades necesarias para ser socialmente competentes) han sido bien documentadas. Además, según estos autores, existiría una conexión entre los déficit en variables cognitivas, biológicas y neuroanatómicas que aparecen en la esquizofrenia y los mostrados en la esquizotipia, de manera que si se estudiasen las habilidades sociales de las personas con esquizotipia podría revelarse esta relación también a nivel de conducta interpersonal. Para comprobar dicha hipótesis, los citados autores realizaron un estudio con sujetos esquizotípicos y comprobaron su falta de habilidad para reconocer emociones en los demás, mostrarse socialmente competentes y seleccionar las conductas apropiadas a cada situación. Los resultados indicaron que estos sujetos presentan las mismas habilidades sociales deficientes que los sujetos con esquizofrenia. Por tanto, esto influirá en sus relaciones interpersonales, de manera que los sujetos con esquizotipia tendrán problemas para desenvolverse en su medio social.

Según Millon y Everly (1985), el estudio de este tipo de variables socioambientales en esquizotipia está justificado por la importancia que tienen los factores ambientales en la etiología y desarrollo de la enfermedad, ya que el aislamiento social de estos sujetos, la sobreprotección por parte de la familia y su conducta dependiente reforzada, además de su tendencia a aislarse de estímulos externos, puede influir en el pronóstico de la enfermedad. Vista la importancia de estas variables en los esquizotípicos clínicos, sería interesante ver hasta que punto son influyentes en el desarrollo de la enfermedad, en aquellos individuos que son identificados como esquizotípicos psicométricos.

Se han elaborado diversos instrumentos de evaluación de la esquizotipia psicométrica, (a diferencia de la esquizotipia genética, en la psicométrica no hay antecedentes familiares de trastorno psicótico, con lo que la posible vulnerabilidad al espectro no tiene componentes genéticos de relevancia) cuyo objetivo es identificar ciertas peculiaridades perceptivas, cognitivas y experienciales que, supuestamente, guardan alguna relación con la sintomatología esquizofrénica y que podrían ser precursoras del riesgo de manifestar trastornos de dicho espectro en sujetos vulnerables. Uno de los más recientes es el Oxford Liverpool Inventory of Feelings and Experiencies (O-LIFE) de Mason, Claridge y Jackson (1995), que mide la esquizotipia a través de cuatro subescalas distintas: Experiencias inusuales, Desorganización cognitiva, Anhedonia introvertida y No conformidad impulsiva. Este instrumento ha demostrado una adecuada fiabilidad y validez en la medida global y de sus diversos componentes (Burch, Steel y Hemsley, 1998).

Derivado de todo lo anterior, el objetivo principal del presente trabajo es estudiar, en población normal, la posible existencia de diferencias emocionales y/o socioambientales entre aquellos individuos con puntuaciones extremas 
en el O-LIFE y en cualquiera de sus subescalas (Esquizotipia Total, Experiencias Inusuales, Desorganización Cognitiva, Anhedonia Introvertida y No Conformidad Impulsiva), es decir, en sujetos esquizotípicos psicométricos.

\section{MÉTODO}

\section{Participantes}

En el estudio participaron 256 estudiantes universitarios, todos ellos estudiantes de $1 .^{\circ} \mathrm{y}$ 2. ${ }^{\circ}$ curso de Psicología en la Universidad de Jaén, de los cuales fueron seleccionados 123 en función de su puntuación: alta (por encima del centil 95) o baja (por debajo del centil 5) en alguna de las cuatro subescalas que forman el O-LIFE y/o en la puntuación total del mismo. Después de esta selección previa, hubo una muerte experimental del 18,7\% (21 de los sujetos abandonaron el estudio antes de concluirlo y otros 2 fueron eliminados por tener antecedentes familiares psicóticos o diferentes trastornos de la personalidad); por tanto, la muestra de sujetos con la que finalmente se realizó el estudio fue de 100 sujetos. Los participantes eran de ambos sexos y sus edades estaban comprendidas entre 18 y 24 años. El número de sujetos en función de cada subescala quedó distribuido del siguiente modo: (1) Experiencias inusuales, 12 sujetos en alta y 12 sujetos en baja. (2) Desorganización cognitiva, 13 sujetos en alta y 10 sujetos en baja. (3) Anhedonia introvertida, 14 sujetos en alta y 21 sujetos en baja. (4) No conformidad impulsiva, 13 sujetos en alta y 11 sujetos en baja. Y (5) Total, 12 sujetos en alta esquizotipia y 11 sujetos en baja esquizotipia. Puesto que alguno de los sujetos pertenecía a más de un grupo, la suma total es superior a 100 .

\section{Pruebas psicológicas}

Oxford Liverpool Inventory of Feelings and Experiencies (O-LIFE; Mason et al., 1995). Empleamos la versión validada con población española (Paíno et al., 1997). Este es uno de los autoinformes recientemente desarrollados para medir esquizotipia y cuya validez y fiabilidad han sido suficientemente demostradas (Burch et al., 1998). Se creó a partir del Combined Schizotypal Traits Questionnaire (CSTQ) de Bentall, Claridge y Slade (1989), que es una combinación de 15 escalas, agrupadas en cuatro factores, que miden por separado diferentes aspectos de la esquizotipia. El O-LIFE está formado por cuatro subescalas derivadas de los cuatro factores de las escalas del CSTQ. Contiene 159 items que hacen referencia a los pensamientos, sensaciones, experiencias y preferencias. El sujeto tiene que marcar con una cruz «Si» o «No» en el lugar numerado correspondiente de la hoja de respuestas, según lo que más se ajuste a su situación. Las cuatro subescalas del O-LIFE son: (1) Experiencias inusuales (EI); Consta de 30 items relacionados con pensamiento mágico, experiencias perceptivas y alucinatorias, consistentes con los síntomas positivos de la psicosis. (2) Desorganización cognitiva (DC); son 24 items que describen dificultades en atención, concentración y toma de decisiones, además de falta de sentido en la vida y ansiedad social. (3) Anhedonia introvertida (AI); 27 items que describen la falta de emociones positivas en las relaciones sociales. Como características fundamentales están la ausencia de placer en la intimidad emocional y física y el énfasis en la independencia y soledad. Y (4) No conformidad impulsiva (NCI); consta de 22 items que recogen información sobre las características desinhibidas y dirigidas por impulsos, haciendo referencia a conductas violentas o excesivas.

Inventario de Depresión de Beck BDI (versión española de Conde y Franch, 1984). Este inventario consta de 19 items que miden depresión, sobre todo los aspectos cognitivos y emocionales del constructo. La puntuación total del sujeto será la suma de los items, cuya valoración se lleva a cabo a través de una escala que va de 0 a 3.

Cuestionario de Ansiedad Estado/Rasgo STAI (Spielberger, Gorsuch y Lushene, 1970) (versión española de TEA, 1982). Esta es una prueba de autoevaluación de la ansiedad como estado transitorio (ansiedad estado) y como rasgo latente (ansiedad rasgo). Consta de dos par- 
tes, con 20 items cada una. La primera evalúa la ansiedad estado y la segunda la ansiedad rasgo.

Escala de Hostilidad (Ho) (Cook y Medley, 1954). Consta de 50 frases en las que el sujeto tiene que decidir, si aplicadas a sí mismo, la frase es verdadera o falsa, según la opción que considere que le describe mejor en términos generales. Habitualmente se utiliza como un indicador general del constructo hostilidad/ira/agresión (HIA), ya que tampoco se ha encontrado una estructura factorial clara en la escala. No obstante, en ocasiones se ha considerado que constituye más bien una medida del componente cognitivo de la emoción de ira. Barefoot, Dodge, Peterson, Dahlstrom y Williams (1989) han propuesto 6 subescalas dentro de la Ho: cinismo, sentimiento hostil, respuesta agresiva, atribución hostil, evitación social y otros, basándose en una propuesta racional. De entre estas subescalas, en nuestro caso se han elegido aquellas que se consideraban más representativas para la personalidad esquizotípica, de acuerdo con sus propias características. Estas han sido: cinismo, que se refiere a una creencia negativa acerca de la naturaleza humana en general, y atribución hostil, que se refiere a la creencia de que las demás personas constituyen una fuente de amenaza. No se ha usado la subescala de evitación social, que es uno de los elementos más importantes de la esquizotipia, porque dicha subescala no se refiere al aislamiento social característico de estos sujetos, sino más bien a la evitación de determinadas personas o situaciones.

Entrevista Estructurada Escrita para la Evaluación de Variables Socio-ambientales. Este instrumento fue elaborado específicamente para la realización de esta investigación. Está formado por 21 items, referidos al ámbito socioambiental del sujeto. Los items han sido divididos en las cinco áreas o subescalas siguientes: (a) Ámbito interpersonal, formado por 2 items. El primero es una escala de valoración en la que el sujeto tiene que indicar la importancia que le da a los siguientes aspectos: familia, estudios, amigos, pareja, trabajo y ocio. El segundo ítem pide a los sujetos que describan acontecimientos vitales relevantes ya sean positivos o negativos. La cantidad se refiere al número de acontecimientos vitales que describen y la calidad a si estos son positivos o negativos. (b) Ámbito familiar, formado por 3 items, que hacen referencia a los siguientes aspectos: relación con los hermanos, número de hermanos, lugar ocupado entre ellos, si viven los dos padres, si los padres viven juntos, relación entre los padres, relación entre hijo y padres (buena, mala, buena con uno y mala con otro) y, por último, la situación económica familiar. (c) Ámbito de apoyo personal percibido, formado por 7 items, donde se evalúan los siguientes aspectos: cambio de residencia, vivienda durante el curso, relación con las personas con las que se convive, disposición de un lugar para estar sólo donde se vive, tiempo que se pasa en este lugar, actividades que se realizan en el mismo, actividades realizadas en el tiempo libre, si el sujeto dispone de pareja, satisfacción con la pareja, días que se está con la pareja, tiempo que se pasa con la pareja, si tiene amigos en Jaén y si pertenece a alguna asociación o colectivo. (d) Ámbito educativo y laboral, formado por 3 items, que aluden a: número de horas de estudio, porcentaje de horas de estudio aprovechadas, si se estudia sólo o acompañado, si tiene trabajo, tiempo que dedica al trabajo a la semana y si el trabajo es remunerado o no. Y (e) Ámbito clínico, formado por 6 items; sólo fue utilizado como criterio excluyente, para descartar del estudio a los sujetos que tuviesen antecedentes familiares de trastornos psicóticos.

\section{Procedimiento}

En primer lugar, se dieron a conocer los objetivos del estudio a los estudiantes de $1 .^{\circ} \mathrm{y}$ 2. ${ }^{\circ}$ de Psicología, solicitándoles su colaboración voluntaria en el estudio. Los sujetos que quisieron colaborar realizaron el O-LIFE durante el horario de clase. Posteriormente, se realizó la selección de submuestras extremas en las puntuaciones de las cuatro subescalas del O-LIFE y/o en la puntuación total del mismo. Los sujetos seleccionados realizaron, de forma voluntaria y colectiva las pruebas STAI, BDI, Entrevista Estructurada Escrita para la Evaluación de Variables Socioambientales, y Ho, en las 
dependencias del departamento de Psicología de la Universidad de Jaén.

\section{RESULTADOS}

Calculado el coeficiente de kurtosis para todas las variables, se comprobó que gran parte de ellas no presentaba una distribución normal, ni tampoco se cumplían los criterios de homoscedasticidad, entre la mayoría de ellas, según la prueba de Barlett. Debido a esto, se optó por la realización de pruebas de análisis no paramétricas. Para variables cuantitativas y ordinales se utilizó la prueba de Mann-Whitney para hacer las comparaciones entre los dos grupos, los de alta y baja esquizotipia (o cualquiera de las subdimensiones que mide el O-LIFE y que también se dividieron en alta y baja). Puesto que una gran mayoría de las características incluidas en las variables socioambientales son nominales $\mathrm{u}$ ordinales, el tipo de análisis que se ha utilizado para evaluar las diferencias entre los grupos fue el coeficiente chi-cuadrado $\left(\chi^{2}\right)$ para las variables nominales y el coeficiente de correlación de Spearman $(\rho)$ para las ordinales. En este último caso, las subescalas no se contemplaron como dicotomizadas, sino que se incluyeron todos los valores (variable continua cuantitativa), ya que de otra forma sería imposible calcular dicho coeficiente. En resumen, las correlaciones entre las variables fueron calculadas usando los coeficientes de Pearson (para relacionar variables cuantitativas entre sí), Spearman (para relacionar variables ordinales con cuantitativas), y el coeficiente $\chi^{2}$ (para relacionar variables ordinales con dicotómicas o dicotomizadas (los grados de libertad de este coeficiente están indicados entre paréntesis).

\section{Diferencias entre los sujetos de alta y baja esquizotipia (puntuación global)}

En primer lugar, fueron analizadas las diferencias en las variables emocionales entre sujetos con alta y baja esquizotipia. Como se puede ver en la Tabla 1, los análisis realizados indicaron que aparecían diferencias significativas entre ambos grupos de sujetos en depresión $(U=0 ; p<0,01)$, ansiedad estado $(U=5$; $p<0,01)$, ansiedad rasgo $(U=5,5 ; p<0,01)$; hostilidad $(U=0 ; p<0,01)$, cinismo $(U=11$; $p<0,01)$ y atribución hostil $(U=8 ; p<0,01)$, obteniendo los sujetos altos en esquizotipia una mayor puntuación que los bajos (véase la Tabla 1)

Posteriormente, se analizaron las diferencias entre los sujetos de alta y baja esquizotipia en las variables socioambientales. El estudio de estas variables se ha dividido en distintos ámbitos: ámbito interpersonal, ámbito familiar, ámbito de apoyo personal percibido y ámbito educativo y laboral. Los análisis realizados en relación con el ámbito interpersonal indican que los sujetos con puntuaciones altas en esquizotipia le dan menos importancia a la familia (obteniendo en este cuestionarios una media de 1,75 en comparación con el grupo de baja esquizotipia, cuya media es de 1,09), siendo la diferencia entre ambos grupos significativa ( $\rho$ $=0,29 ; p<0,01)$. También aparecen diferencias, como se puede ver en la Tabla 2 , en la cantidad y calidad de los acontecimientos vitales, habiendo una mayor proporción de sujetos que consideran que han sufrido acontecimientos vitales $\left(\chi_{(1)}^{2}=3,7 ; p<0,05\right)$ y considerando estos más negativos $\left(\chi_{(4)}^{2}=8,139 ; p=0,08\right.$, diferencia marginalmente significativa) en el

Tabla 1. Medias (y desviaciones típicas) de las variables emocionales en sujetos con alta y baja esquizotipia

\begin{tabular}{lcc}
\hline Variable emocional & $\begin{array}{c}\text { Altos en esquizotipia } \\
(n=12)\end{array}$ & $\begin{array}{c}\text { Bajos en esquizotipia } \\
(n=11)\end{array}$ \\
\hline Depresión & $17,67(7,25)$ & $1,00(1,00)$ \\
Ansiedad estado & $29,92(11,14)$ & $9,73(4,96)$ \\
Ansiedad rasgo & $34,50(11,71)$ & $9,64(5,02)$ \\
Hostilidad & $30,00(6,36)$ & $11,64(3,44)$ \\
Cinismo & $7,58(2,80)$ & $3,27(1,95)$ \\
Atribución hostil & $8,58(1,50)$ & $5,19(1,53)$ \\
\hline
\end{tabular}


Tabla 2. Calidad y cantidad de acontecimientos vitales (AV) en sujetos altos y bajos en esquizotipia

\begin{tabular}{lll}
\hline Variables ámbito interpersonal & \multicolumn{1}{c}{$\begin{array}{c}\text { Altos en esquizotipia } \\
(n=12)\end{array}$} & \multicolumn{1}{c}{$\begin{array}{c}\text { Bajos en esquizotipia } \\
(n=11)\end{array}$} \\
\hline Cantidad de acontecimientos vitales & $\begin{array}{l}83,3 \%(10 \text { de } 12 \text { sujetos declaran } \\
\text { tener AV })\end{array}$ & $\begin{array}{l}45,5 \% \text { (5 de 11 sujetos } \\
\text { declaran tener AV) }\end{array}$ \\
Calidad de acontecimientos vitales & $\begin{array}{l}\text { Negativos para el 70 \% de los } \\
\text { sujetos (para 7 de 10 sujetos) }\end{array}$ & $\begin{array}{l}\text { Negativos para el 20 } \% \text { de los } \\
\text { sujetos (para 1 de 5 sujetos) }\end{array}$ \\
\hline
\end{tabular}

grupo de alta que en el grupo de baja esquizotipia.

Los análisis realizados en las áreas familiar y de apoyo social percibido indicaron que no existían diferencias significativas en ninguna de las variables medidas entre los sujetos de alta y baja esquizotipia. Por último, se llevó a cabo el análisis del ámbito educativo y laboral. En este caso, los análisis mostraron que sólo aparecían diferencias significativas en el porcentaje de horas de estudio aprovechadas $(U=$ $35,5 ; p<0,05)$, presentando los sujetos con alta esquizotipia un porcentaje menor $(58,73 \%)$ que los de baja esquizotipia $(74,84 \%)$.

\section{Diferencias entre los sujetos altos y bajos en cada una de las dimensiones del O-LIFE}

Encontramos diferencias significativas $(p<$ 0,01 ) en todas las variables emocionales entre los sujetos altos y bajos en cada una de las dimensiones del O-LIFE. Así, las diferencias entre los sujetos con puntuación alta y baja en Experiencias inusuales (EI) aparecen en depresión $(U=9,5)$, ansiedad estado $(U=18,5)$, ansiedad rasgo $(U=14,5)$, hostilidad $(U=6)$, cinismo $(U=20)$ y atribución hostil $(U=11,5)$, presentando los sujetos con alta puntuación en EI mayores puntuaciones en todas las variables que los sujetos con baja puntuación en dicha subescala. En la subescala de Desorganización cognitiva (DC) se pueden observar igualmente diferencias significativas $(p<0,01)$ en depresión $(U=0)$, ansiedad estado $(U=19,5)$, ansiedad rasgo $(U=2)$, hostilidad $(U=0)$, cinismo $(U=20)$ y atribución hostil $(U=14)$, presentando los sujetos con alta DC una puntuación mayor que los de baja DC en todas las variables emocionales. Por su parte, en la subescala Anhedonia introvertida (AI) se encuentran diferencias significativas en depresión $(U=54,5 ; p<$ $0,01)$, ansiedad estado $(U=79 ; p<0,05)$, ansiedad rasgo $(U=47 ; p<0,01)$, hostilidad $(U=$ $51,5 ; p<0,01)$, cinismo $(U=89 ; p<0,05)$ y atribución hostil $(U=89 ; p<0,05)$, con una puntuación mayor en todas las variables en los sujetos de alta AI que en los de baja AI. Por último, en la subescala de No Conformidad impulsiva (NCI) los sujetos con puntuación alta presentan igualmente puntuaciones mayores que los de baja NCI en depresión $(U=22 ; p<0,01)$, ansiedad estado $(U=36,5 ; p<0,05)$, hostilidad $(U=19 ; p<0,01)$, cinismo $(U=33,5 ; p<0,05)$ y atribución hostil $(U=32 ; p<0,05)$.

En cuanto a las variables socioambientales, se han hallado también diferencias estadísticamente significativas dependiendo de la subescala y la variable medida. Respecto a la subescala de Experiencias inusuales, los resultados muestran una relación positiva con los dos elementos pertenecientes al ámbito interpersonal. En este sentido, la escala se ha relacionado con las variables familia - se toman las puntuaciones de la subescala como puntuaciones cuantitativas- $(\rho=0,34 ; p<0,01)$ y acontecimientos vitales - se toman las puntuaciones de la subescala como puntuaciones nominales $\left(\chi_{(1)}^{2}\right.$ $=5,04 ; p<0,05)$ - En relación con la primera variable, los resultados muestran que a mayor puntuación en esta escala menor importancia es otorgada a la familia. En cuanto a la segunda, parece que los sujetos con alta puntuación en la subescala sufren mayor número de acontecimientos vitales que los de baja puntuación (91,67\% frente al 55,55\%).

También se han hallado algunas relaciones y diferencias significativas entre los sujetos con 
Tabla 3. Medias (y desviaciones típicas) de las variables emocionales en los sujetos altos y bajos en las subescalas del O-LIFE

\begin{tabular}{|c|c|c|c|c|c|c|c|c|}
\hline & \multicolumn{2}{|c|}{$\begin{array}{l}\text { Experiencias } \\
\text { inusuales }\end{array}$} & \multicolumn{2}{|c|}{$\begin{array}{c}\text { Desorganización } \\
\text { cognitiva }\end{array}$} & \multicolumn{2}{|c|}{$\begin{array}{l}\text { Anhedonia } \\
\text { introvertida }\end{array}$} & \multicolumn{2}{|c|}{$\begin{array}{c}\text { No conformidad } \\
\text { impulsiva }\end{array}$} \\
\hline & $\begin{array}{c}\text { Altos } \\
(n=12)\end{array}$ & $\begin{array}{c}\text { Bajos } \\
(n=12)\end{array}$ & $\begin{array}{c}\text { Altos } \\
(n=13)\end{array}$ & $\begin{array}{c}\text { Bajos } \\
(n=10)\end{array}$ & $\begin{array}{c}\text { Altos } \\
(n=14)\end{array}$ & $\begin{array}{c}\text { Bajos } \\
(n=21)\end{array}$ & $\begin{array}{c}\text { Altos } \\
(n=13)\end{array}$ & $\begin{array}{c}\text { Bajos } \\
(n=11)\end{array}$ \\
\hline & $14,9 \quad(6,3)$ & $3,2(4,1)$ & $15,1 \quad(8,2)$ & $1,0(1,1)$ & $16,4 \quad(7,9)$ & $7,3 \quad(7,6)$ & $14,7 \quad(7,13)$ & $6,4 \quad(5,3)$ \\
\hline Ansiedad-estado & $24,7(10,7)$ & $11,2(4,7)$ & $26,4(14,1)$ & $9,6($ & $25,9(13,8)$ & $15,9(11,0$ & $24,6(12,0)$ & $15,8(10,0)$ \\
\hline Ansiedad-rasgo & $30,3(11,9)$ & $12,5(5,5)$ & $33,9 \quad(8,5)$ & 7,5 & $33,6 \quad(9,3)$ & $18,7(12,0)$ & $25,7(14,2)$ & $22,3 \quad(9,0)$ \\
\hline Hostilidad & $30,7 \quad(6,9)$ & $14,4(7,0)$ & $28,6 \quad(7,07)$ & $12,3(5,4)$ & $27,8 \quad(7,64)$ & $17,0 \quad(6,5)$ & $27,3 \quad(5,0)$ & $17,1 \quad(6,0)$ \\
\hline Cinismo & $8,3 \quad(2,9)$ & $4,1(3,2)$ & $7,6 \quad(3,8)$ & $3,8(2,6)$ & $8,2 \quad(3,26)$ & $5,6 \quad(3,2)$ & $8,1 \quad(2,5)$ & $5,5 \quad(3,3)$ \\
\hline Atribución hostil & $8,8 \quad(1,5)$ & $5,6(1,8)$ & $7,9 \quad(1,9)$ & $5,2(1,3)$ & $8,0 \quad(1,71)$ & $6,6 \quad(1,9)$ & $7,9 \quad(1,8)$ & $6,4 \quad(1,3)$ \\
\hline
\end{tabular}

puntuaciones altas y bajas en la subescala Desorganización cognitiva, tanto en el ámbito familiar, como en el educativo y laboral. En este caso, las variables implicadas han sido «lugar ocupado entre los hermanos» $(\rho=0,26$; $p<0,05)$ y «porcentaje de horas de estudio aprovechadas» $(U=16,5 ; p<0,01)$, siendo el lugar que los sujetos con puntuaciones altas en la subescala ocupa entre los hermanos posterior $(2,75$ frente a 1,9$)$ y menor el porcentaje de horas de estudio aprovechadas $(57,17 \%$ frente a $82,15 \%$ ) que lo mostrado por los sujetos con puntuaciones bajas en esta subescala.

Por su parte, en la subescala Anhedonia introvertida se encuentran también algunas asociaciones y diferencias estadísticamente significativas, entre los grupos alto y bajo, en dos variables del ámbito familiar: concretamente, en las variables «número de hermanos» $(U=$ $200,5 ; p<0,05)$ y «lugar ocupado entre los hermanos» $(\rho=0,35 ; p<0,01)$, caracterizándose los sujetos con alta AI por tener mayor número de hermanos (3 frente a 2,32) y siendo el lugar que ocupan entre los hermanos posterior $(2,38$ frente a 1,32) que los sujetos con puntuaciones bajas en AI.

Por último, en la subescala No conformidad impulsiva se han encontrado asociaciones y diferencias significativas entre sujetos altos y bajos en relación con algunas variables de los ámbitos interpersonal, familiar, y educativolaboral. En el primero de ellos, se encuentran relaciones con las variables familia $(\rho=0,36$; $p<0,01)$, pareja $(\rho=0,21 ; p<0,05)$ y acontecimientos vitales $\left(\chi_{(1)}^{2}=4,11 ; p<0,05\right)$. Así, cuanto más alta es la puntuación en la subescala, menor importancia se da a la familia y a la pareja y mayor número de acontecimientos vitales se sufren (91,67\% frente al 45,45\%). En el segundo de ellos, se hallan diferencias con las variables «viven juntos los padres» $\left(\chi_{(1)}^{2}=\right.$ $4,062 ; p<0,05)$, «relación entre los padres» $\left(\chi_{(1)}^{2}=8,362 ; p<0,01\right)$ y «relación entre hijo $\mathrm{y}$ padres» $\left(\chi_{(2)}^{2}=8,362 ; p<0,05\right)$. En concreto, parece que hay más padres separados $(84,62 \%$ frente al 100\%) y una peor relación en aquellos padres que están juntos $(61,53 \%$ frente al $100 \%$ ) en los sujetos con puntuaciones altas en la subescala frente a los que tienen una puntuación baja en la misma; además, los sujetos con puntuaciones altas también se llevan peor con sus padres frente a los que muestran puntuaciones bajas $(53,84 \%$ frente al $100 \%)$. En cuanto al ámbito educativo y laboral, los resultados exhiben diferencias significativas entre sujetos altos y bajos en esta subescala en la variable porcentaje de horas de estudio aprovechadas $(U=37$; $p<0,05$ ), siendo menor el porcentaje de horas de estudio aprovechadas en los sujetos con puntuaciones altas frente a los que tienen puntuaciones bajas $(58,03 \%$ frente a $69,87 \%)$.

\section{DISCUSIÓN}

Diferencias entre los sujetos con alta y baja esquizotipia (medida global)

Los resultados de este estudio muestran que existen diferencias estadísticamente significa- 
tivas entre los dos grupos de sujetos en todas las variables que han sido medidas a nivel emocional, presentando mayores puntuaciones los sujetos de alta esquizotipia en depresión, ansiedad estado, ansiedad rasgo, hostilidad, cinismo y atribución hostil (constituyendo éstas tres últimas las medidas cognitivas de la emoción de ira). En otros estudios (Lenzenweger et al., 1991; Wolfradt y Straube, 1998), al igual que en esta investigación, los altos niveles de depresión y de ansiedad han sido relacionados con puntuaciones altas en esquizotipia psicométrica. Wolfradt y Straube (1998) los consideran signos tempranos de vulnerabilidad. Para autores como Kendler (1985) y Meehl (1962) la ansiedad es considerada un rasgo importante de los esquizotípicos genéticos $\mathrm{y}$, como se puede comprobar, también es importante dentro de la esquizotipia psicométrica.

En lo que se refiere a la hostilidad, hay autores (Eurelings-Bontekoe et al., 1997) que consideran que las personas con trastornos de la personalidad tienden a ser hostiles. Para estos autores, la hostilidad sería el mayor predictor independiente de patología de la personalidad. A este respecto, Shean y Wais (2000) encontraron que los sujetos con altas puntuaciones en esquizotipia genética mostraban más hostilidad que los sujetos con baja puntuación. Estos mismos resultados han sido confirmados en esta investigación, tomando medidas de esquizotipia psicométrica; en concreto, se ha encontrado que los sujetos con alta esquizotipia psicométrica muestran también puntuaciones más altas en hostilidad. Con respecto al cinismo y la atribución hostil, en función de los resultados de esta investigación, se comprueba que en los esquizotípicos piscométricos estas características aparecen con mayor intensidad que en los sujetos de baja esquizotipia. Este dato es novedoso, por lo que habría que seguir investigando sobre el tema para confirmarlo.

La alta carga emocional negativa encontrada en los sujetos con alta esquizotipia (diferencias entre los grupos en todas las variables emocionales medidas) podría ser la base para explicar, en parte, las alteraciones de las relaciones sociales e interpersonales que caracterizan a los sujetos con esquizotipia según el DSM-IV-TR
(APA, 2002). Así, García-León y Reyes del Paso (2002) vinculan la alta puntuación en hostilidad con la aparición de características como la poca cohesión y el poco apoyo social, lo que conduce a gran cantidad de conflictos interpersonales en la población general. Del mismo modo, es aceptable que la mayor tasa de depresión y ansiedad en un sujeto afecte negativamente a las relaciones interpersonales de éste (Adams, 1975). Dado que en todas las variables hay diferencias, podría interpretarse que si el sujeto desarrolla algún tipo de trastorno psicótico en un futuro, los síntomas emocionales van a estar presentes dentro de su cuadro patológico, independientemente del tipo de trastorno que desarrolle y de los demás síntomas que presente. Dicha idea reforzaría la argumentación de Wolfradt y Straube (1998), quienes señalaron las alteraciones emocionales como marcadores de vulnerabilidad al espectro esquizofrénico.

En lo referente a las variables socioambientales, los sujetos con alta esquizotipia psicométrica, en el ámbito interpersonal, dan menos importancia a la familia y un mayor número de ellos declaran haber sufrido hechos valorados como acontecimientos vitales estresantes. Además, también presentan diferencias en el ámbito educativo y laboral, en el porcentaje de horas de estudio aprovechadas, indicando que los de alta esquizotipia aprovechan menos horas de estudio. Todas estas diferencias van a analizarse de manera más detallada.

Estas variables estarían relacionadas con los problemas a nivel interpersonal que son característicos de los esquizotípicos genéticos y, por tanto, sería interesante comprobar la relevancia que dichas variables tienen en los sujetos esquizotípicos psicométricos. El hecho de que los sujetos que puntúan alto en esquizotipia otorguen menos importancia a la familia, parece contradecir la abundante literatura sobre la dependencia a la familia que muestran los esquizofrénicos. No obstante, dicha contradicción podría explicarse teniendo en cuenta que los individuos con una alta puntuación en esquizotipia no muestran los síntomas propios de la enfermedad y, por tanto, es posible que esa dependencia no exista. También podría explicarse este resultado haciendo hincapié en 
el significado de dependencia familiar: aunque el esquizotípico fuese dependiente de su familia (como lo es el esquizofrénico), esta dependencia puede no corresponder con la importancia subjetiva que el sujeto le concede a su familia.

También existe un mayor porcentaje de sujetos que consideran que han sufrido acontecimentos vitales (y, marginalmente, éstos son más negativos) en el grupo de alta esquizotipia psicométrica. Estos datos son aparentemente inexplicables ya que no es esperable que, por el mero hecho de obtener una puntuación determinada en un cuestionario, le sucedan a una persona más o menos acontecimientos vitales. Sin embargo, podrían apuntarse dos explicaciones. Por un lado, que en realidad el mismo número de sujetos padezca acontecimientos vitales tanto en alta como en baja esquizotipia (que sería lo esperable), pero que aquellas personas con baja esquizotipia no les den la relevancia que sí les dan las personas con alta esquizotipia (que son más susceptibles emocionalmente hablando, como lo prueban los datos obtenidos en esta investigación), con lo que aparentemente, más sujetos con alta esquizotipia padecerían acontecimientos vitales. Esta hipótesis se vería apoyada por el trabajo de Brown, Birley y Wing (1972), que afirmaban que el enfermo esquizofrénico es extremadamente sensible al ambiente social (por lo que su nivel de activación es fácilmente desbordado por aquél), y por otros estudios que han constatado una estrecha relación entre la percepción del estrés vital y diversos trastornos y/o síntomas psicopatológicos (Sanjuán y Magallares, 2006; Sandín, Rodero, Santed y García-Campayo, 2006). Por otro lado, una segunda explicación vendría dada si se acepta que en realidad es cierto que mayor número de sujetos con alta esquizotipia sufren acontecimientos vitales en comparación con los de baja esquizotipia. Para admitir esta afirmación sería necesario un análisis más profundo de los acontecimientos vitales que declaran haber padecido los sujetos de este estudio, con el fin de encontrar posibles acontecimientos vitales no fortuitos sino provocados en parte por el propio sujeto, que explicarían las diferencias entre grupos. Así, no es esperable que haya diferencias entre alta y baja esquizotipia en ocurrencias de acontecimientos vitales como muertes de familiares o accidentes domésticos (que son fortuitos), sin embargo, dada la dificultad en las interacciones sociales de estos sujetos, es posible que algunos de esos acontecimientos vitales fuesen provocados por los propios sujetos (peleas, discusiones, rupturas de pareja, etc.). A causa de lo escueto de las respuestas de los sujetos a este ítem no es posible profundizar en este sentido (un análisis reciente sobre sucesos vitales y psicopatología puede verse en Talarm, Navarro, Rossell y Rigat, 2006).

Respecto a la calidad de los acontecimientos vitales, el hecho de que los sujetos con alta esquizotipia presenten (marginalmente) mayor número de acontecimientos vitales negativos podría explicarse también en la línea de la primera hipótesis planteada: dar mayor peso subjetivo a los aspectos negativos de los acontecimientos que les suceden. En este sentido, es posible que los sujetos con alta esquizotipia tengan puntuaciones más elevadas en un constructo propuesto en las últimas décadas y relacionado con los estados emocionales, como es el de emotividad negativa (Sandín et al., 1999). Éste se define como la disposición a experimentar estados emocionales aversivos y a tener una visión negativa de sí mismo y del mundo. Se ha relacionado tanto con la ansiedad como con la depresión y la ira (Watson, Clark y Carey, 1988), variables que, como se ha visto, presentan los sujetos con alta esquizotipia. También para confirmar esta hipótesis sería necesario realizar nuevas investigaciones.

A nivel educativo, los resultados indican que los sujetos que puntúan alto en esquizotipia tienen un porcentaje menor de horas de estudio aprovechadas que los sujetos que puntúan bajo en esquizotipia. Una posible explicación a este hecho podría venir dada por los problemas atencionales que muestran estos sujetos (Jiménez, Muela, García-León y Garrancho, 2004), ya que al tener un déficit de atención sostenida (Lenzenweger et al., 1991), es posible que les suponga un mayor esfuerzo concentrarse en la realización de una tarea durante un tiempo prolongado como es el estudio. 


\section{Diferencias entre los sujetos altos y bajos en las dimensiones del O-LIFE}

En las variables emocionales que han sido medidas aparecen diferencias en todas las subescalas entre los grupos de alta y baja puntuación, como sucede en otros estudios anteriores en los que se utilizó también el O-LIFE (Lewandowski et al., 2006). Es decir, todos los sujetos que puntúan alto en cada subescala muestran mayores niveles de depresión, ansiedad rasgo y estado, hostilidad, cinismo y atribución hostil. La única excepción la constituye la subescala de No conformidad impulsiva, en la que no han aparecido diferencias en la ansiedad rasgo entre los grupos con altas y bajas puntuaciones en NCI. Es probable que esto sea debido a que la ansiedad no es una característica que aparezca en estos sujetos, que se guían por conductas desinhibidas y dirigidas por impulsos, sin pensar en las consecuencias que sus actos pueden producir. Parece lógico pensar que la ansiedad aparezca cuando se piensa en las consecuencias de los actos que se realizan, justamente lo contrario de lo que podría suceder en este caso. No obstante, dado que en las demás variables emocionales sí aparecen diferencias entre los grupos dentro de esta subescala, se puede afirmar que todas las subescalas son sensibles a las diferencias emocionales entre alta y baja esquizotipia. Estas variables emocionales, posiblemente por lo apuntado anteriormente respecto a la alta carga emocional en los sujetos con alta esquizotipia, podrían explicar en parte las alteraciones a nivel de relaciones sociales e interpersonales características de estos sujetos, según el DSM-IV-TR (APA, 2002). Dado que aparecen diferencias en prácticamente todas las variables emocionales en todas las subescalas, podría predecirse (como ya se hizo al comentar los resultados emocionales en la escala total) que si el sujeto desarrollase con el tiempo algún tipo de trastorno psicótico, los síntomas emocionales iban a estar presentes en su cuadro patológico, y que esto reforzaría la idea que interpreta las alteraciones emocionales como marcadores de vulnerabilidad al espectro. Además, tal y como afirman Lewandowski et al. (2006), es importante prestar una gran atención a los síntomas de depresión y ansiedad en la esquizotipia, puesto que estos síntomas podrían ser buenos indicadores de un incremento en el riesgo de desarrollar psicosis. Sería interesante resaltar las altas puntuaciones de hostilidad encontradas en este estudio en sujetos con puntuaciones altas en esquizotipia psicométrica y/o en sus distintas subescalas. En concreto, estos sujetos presentan mayor carga de hostilidad y al mismo tiempo problemas en el ámbito interpersonal. Habría que realizar un estudio más extenso en el que se mida también el apoyo social de los esquizotípicos psicométricos para poder llegar a presentar alguna conclusión a este respecto.

Centrándonos en las variables socioambientales que aparecen relacionadas con las distintas subescalas del O-LIFE, en todas las subescalas aparece alguna relación con alguno de los ámbitos en los que se han dividido las variables socioambientales. Estos hallazgos referidos a las subescalas son novedosos en la literatura. $\mathrm{La}$ subescala Experiencias inusuales ha aparecido relacionada con las variables familia y acontecimientos vitales, es decir, con el ámbito interpersonal socioambiental. Los sujetos con altas puntuaciones en la subescala dan menos importancia a la familia y, además, sufren más acontecimientos vitales que los que puntúan bajo en la escala.

Las diferencias en la dimensión Desorganización cognitiva aparecen en las variables «lugar ocupado entre hermanos» y en el «porcentaje de horas de estudio aprovechadas». Los sujetos que puntúan alto en esta dimensión son los más jóvenes dentro de sus familias, y además, el porcentaje de sus horas de estudio aprovechadas es menor. El hecho de que estos sujetos sean, mayoritariamente, los menores entre los hermanos hace necesario que en un futuro se estudien en profundidad las relaciones entre hermanos (rivalidad, envidia, sobreprotección...). Por su parte, el bajo porcentaje de horas de estudio aprovechadas que declaran tener se puede explicar por los problemas de atención que sufren, ya que los sujetos que puntúan alto en esta subescala también tienen problemas en atención sostenida (Jiménez, Muela, García-León y Garrancho, 2004).

En la subescala Anhedonia introvertida las diferencias entre los grupos altos y bajos apa- 
recen en las variables «número de hermanos»y «lugar ocupado entre los hermanos». Los resultados indican que los sujetos que puntúan alto en esta subescala, además de pertenecer a familias más numerosas, son los más jóvenes dentro de las mismas. De forma que es posible que a estos sujetos les ocurra lo mismo que a los que puntúan alto en DC; con lo que lo más adecuado sería profundizar en un futuro en las relaciones familiares de estos sujetos.

En lo que se refiere a la subescala No conformidad impulsiva, las diferencias entre los grupos que han aparecido en los resultados se refieren al ámbito interpersonal (mostrando los sujetos con alta puntuación en la escala más acontecimientos vitales $\mathrm{y}$ dándole menor importancia a la familia) y en el ámbito familiar (entre los sujetos que puntúan alto en la subescala hay más casos de padres separados, peor relación entre los padres y peor relación de los sujetos con los padres). Es posible que un ambiente familiar menos positivo conduzca a una mayor desprotección en estos sujetos y a un aprendizaje más deficiente en lo que respecta a unas adecuadas normas sobre las relaciones interpersonales, lo que, junto con su peor control de los impulsos, les podría llevar a interacciones más negativas y violentas con los demás.

Por último, es importante subrayar que las hipótesis y relaciones entre distintas variables que se han dado para explicar los resultados socioambientales en las cuatro subescalas, deben ser tomadas con precaución y considerarse como meras probabilidades puesto que no hay una base documental previa que investigue estos aspectos, y además los datos, en su mayoría, son cualitativos y se han obtenido mediante autoinformes, por lo que son inadecuados para establecer hipótesis funcionales de causaefecto. En este sentido, sería interesante investigar también dicha relación más profundamente (por ejemplo con instrumentos más objetivos, con mayor tiempo de seguimiento, con muestras más numerosas...), para identificar hasta que punto y de qué modo influyen estas variables en los sujetos con puntuaciones altas en esquizotipia psicométrica.

En resumen, las variables emocionales negativas aparecen ligadas a los sujetos con puntua- ciones altas en esquizotipia psicométrica, mientras que apenas se encuentran en sujetos con puntuaciones bajas en esta variable. En este estudio, los esquizotípicos psicométricos no tienen ningún antecedente familiar que padezca algún trastorno psicótico. Debido a ello, heredarán menores alteraciones cognitivas, al no padecerlas sus progenitores, mientras que podrán mostrar alteraciones emocionales, las cuales tienen mayor influencia ambiental. En este sentido, es probable que si los sujetos con alta puntuación en esquizotipia (y/o en las subescalas de Experiencias Inusuales y No conformidad Impulsiva) llegan a desarrollar algún trastorno en el futuro, y con independencia de cuál sea, éste se caracterizará por incluir elementos emocionales como una parte importante del mismo. Por tanto, la evaluación de estos componentes emocionales es indispensable en dichos sujetos de cara a una prevención precoz. En cuanto a las variables socioambientales, éstas están muy relacionadas igualmente con la esquizotipia psicométrica, sobre todo las referidas al ámbito interpersonal y familiar del sujeto y las referidas al ámbito educativo del mismo. Es posible que los problemas interpersonales y de atención que son característicos en los sujetos esquizotípicos y, que podrían estar causando problemas en el ambiente social del sujeto, sean también marcadores de vulnerabilidad importantes en estas personas.

\section{REFERENCIAS BIBLIOGRÁFICAS}

Adams, G.S. (1970). Medición y evaluación en educación, psicología y "guidance». Barcelona: Herder. (Original en ingles 1964).

American Psychiatric Association (APA) (2002). DSMIV-TR. Manual diagnóstico y estadístico de los trastornos mentales. Texto revisado. Barcelona: Masson. (Original en ingles 2000).

Barefoot, J.C., Dodge, K.A., Peterson, B.L., Dahlstrom, W.G., y Williams, R.B. (1989). The Cook-Medley Hostility Scale: Item content and ability to predict survival. Psychosomatic Medicine, 51, 46-57.

Becoña, E. (2006). Resiliencia: Definición, características y utilidad del concepto. Revista de Psicopatología y Psicología Clínica, 11, 125-146.

Bentall, R.P., Claridge, G.S., y Slade, P.D. (1989). The multidimensional nature of schizotypal traits: A factor 
analytic study with normal subjects. British Journal of Clinical Psychology, 28, 363-375.

Braunstein-Bercovitz, H. (2000). Is the attentional dysfunction in schizotypy related to anxiety? Schizophrenia Research, 46, 255-267.

Brown, G., Birley, J., y Wing, J. (1972). Influence of family life on the course of schizophrenic disorder: A replication. British Journal of Psychiatry, 121, 241258.

Burch, G., Steel, C., y Hemsley, D.R. (1998). OxfordLiverpool Inventory of Feelings and Experiences: Reliability in an experimental population. British Journal of Clinical Psychology, 37, 107-108.

Burman, B., Mednick, S.A., Machón, R.A., Parnas, J., y Schulsinger, F. (1987). Children at high risk for schizophrenia: Parent and offspring perceptions of family relationships. Journal of Abnormal Psychology, 96, 364-366.

Cabrera, P., Urrutia, B., Vera, V., Alvarado, M., y VeraVillarroel, P. (2005). Ansiedad y depresión en niños diagnosticados con cáncer. Revista de Psicopatología y Psicología Clínica, 10, 115-123.

Conde, V., y Franch, J.J. (1984). Escalas de evaluación comportamental para la cuantificación de la sintomatología de los trastornos angustiosos y depresivos. Madrid: Upjohn Farmaquímica.

Cook, W., y Medley, D. (1954). Proposed hostility for pharisaic-virtue skills of the MMPI. Journal of Applied Psychology, 38, 414-418.

Cornblatt, B.A., Lenzenweger, M.F., Dworkin, R.H., y Erlenmeyer-Kimling, L. (1992). Chilhood attentional dysfunctions predict social deficits in unaffected adults at risk for schizophrenia. British Journal of Psychiatry, 161, 59-64.

Edo, M.T., y Ballester, R. (2006). Estado emocional y conducta de enfermedad en pacientes con vih-sida y enfermos oncológicos. Revista de Psicopatología y Psicología Clínica, 11, 79-90.

Eurelings-Bontekoe, E.H.H., Van der Slikke, M., y Verschuur, M.J. (1997). Psychological distress, depressive symptomatology, coping and DSM-III-R/ICD-10 personality disorders. A study among primary mental health care patients. Personality and Individual Differences, 23, 407-417.

Fernández-Montalvo, J., y Echeburúa, E. (2006). Uso y abuso de los autoinformes en la evaluación de los trastornos de personalidad. Revista de Psicopatología y Psicología Clínica, 11, 1-12.

García-León, A., y Reyes del Paso, G.A. (2002). Una revisión de los modelos explicativos de la relación entre el constructo hostilidad-ira-agresión y la enfermedad coronaria. Anales de Psicología, 18, 61-76.

Godoy, J.F. (1990). Avances en la intervención cognitivoconductual en la esquizofrenia. En Junta de Andalucía
(Ed.). Seminario de la Unidad de Docencia y Psicoterapia (pp.70-86). Sevilla: Junta de Andalucía.

Godoy, J.F., Muela, J.A, Sánchez-Barrera, M.B., SánchezHuete, J.R., Pérez, M., Martínez, J.M., Anguiano, I., Lorite, A., y Marí-Beffa, P. (1995). Marcadores de vulnerabilidad/y factores de protección en la prevención de la esquizotipia. Psicología Conductual, 3, 35-46.

Hodges, A., Byrne, M., Grant, E., y Johnstone, E. (1999). People al risk of schizophrenia: sample characteristics of the first 100 cases in the Edinburgh high-risk study. British Journal of Psychiatry, 174, 547-553.

Jiménez, M.D, Muela, J.A., García-León, A., y Garrancho, M.D. (2004). Esquizotipia psicométrica y alteraciones atencionales. Psicothema, 16, 22-26.

Kendler, K.S. (1985). Diagnostic approaches to schizotypal personality disorder: A historical perspective. Schizophrenia Bulletin, 11, 538-553.

Lewandowski, K.E., Barrantes-Vidal, N., Nelson-Gray, R.O., Clancy, C., Kepley, H.O., y Kwapil, T.R. (2006). Anxiety and depression symptoms in psychometrically identified schizotypy. Schizophrenia Research, 83, 225-235.

Lenzenweger, M.F., Cornblatt, B.A., y Putnick, M. (1991). Schizotypy and sustained attention. Journal of Abnormal Psychology, 100, 84-89.

Martínez, P.C., Ferrando, P.J., Lemos, S., Inda, M., Paino P, M., y López, A.M. (1999). Naturaleza y estructura del constructo esquizotipia. Análisis y Modificación de Conducta, 25, 615-637.

Mason, O., Claridge, G., y Jackson, M. (1995). New scales for the assessment of schisotypy. Personality and Individual Differences, 18, 7-13.

Meehl, P. (1962). Schizotaxia, schizotypy, schizophrenia. American Psychologist, 17, 827-838.

Millon, T., y Davis, R. (2006). Trastornos de la personalidad en la vida moderna (2. ${ }^{\mathrm{a}}$ ed.). Barcelona: Masson. (Original en inglés 2004).

Millon, T., y Everly, G.S. (1985). La personalidad y sus trastornos. Barcelona: Martínez Roca. (Original en inglés 1985).

Paíno, M.M., López, A.M., Inda, M., Martínez, P.C., y Lemos, S. (1997). Validación externa de dos cuestionarios de esquizotipia. Psicothema, 9, 175-186.

Sandin, B., Chorot, P., Lostao, L., Joiner, T.E., Santed, M.A., y Valiente, R.M. (1999). Escalas PANAS de afecto positivo y negativo: Validación factorial y convergencia transcultural. Psicothema, 11, 37-51.

Sandin, B., Rodero, B., Santed, M.A., y García-Campayo, J. (2006). Sucesos vitales estresantes y trastorno de pánico: Relación con la ocurrencia del trastorno de pánico, la gravedad clínica y la agorafobia. Revista de Psicopatología y Psicología Clínica, 11, 179-190.

Sanjuán, P., y Magallares, A. (2006). Estilo atributivo negativo, sucesos vitales y sintomatología depresiva. 
Revista de Psicopatología y Psicología Clínica, 11, 9198.

Shean, G., y Wais, A. (2000). Interpersonal behavior and schizotypy. Journal of Nervous and Mental Disease, 188, 842-846.

Spielberger, C.D., Gorsuch, R.L., y Lushene, R.E. (1970). Manual for the State/Trait Anxiety Inventory. Palo Alto: Consulting Psychologists Press (versión española, TEA, 1982).

Straube, E.R., y Oades, R. (1992). Schizophrenia. Empirical research and findings. New York: Academic Press.

Talarn, A., Navarro, N., Rossell, L., y Rigat, A. (2006). Propuesta de especificadores diagnósticos vinculados al estrés y el trauma: Una aportación a la nosología psicopatológica. Revista de Psicopatología y Psicología Clínica, 11, 107-114.

Waldeck, T.L., y Miller, L.S. (2000). Social skills deficits in schizotypal personality disorder. Psychiatry Research, 93, 237-246.

Watson, D., Clark, L.A., y Carey, G. (1988). Positive and negative affect and their relation to anxiety and depressive disorders. Journal of Abnormal Psychology, 97, 346-353.

Wolfradt, U., y Straube E.R. (1998). Factor sturcture of schizotypal traits among adolescents. Personality and Individual Differences, 24, 201-206. 